Current psychology letters

\title{
Visual/ auditory processing and categorization preferences in 5-year-old children and adults
}

Carole Berger and Sophie Donnadieu

\section{(2) OpenEdition \\ 1 Journals}

Electronic version

URL: http://journals.openedition.org/cpl/3673

DOI: $10.4000 / \mathrm{cpl} .3673$

ISSN: $1379-6100$

Publisher

Centre PsyCLÉ

\section{Electronic reference}

Carole Berger and Sophie Donnadieu, « Visual/ auditory processing and categorization preferences in 5-year-old children and adults », Current psychology letters [Online], Vol. 24, Issue 2, 2008 | 2008, Online since 08 September 2008, connection on 08 September 2020. URL : http://journals.openedition.org/ cpl/3673 ; DOI : https://doi.org/10.4000/cpl.3673 
To quote this document:

Carole Berger and Sophie Donnadieu, «Visual/ auditory processing and categorization preferences in 5-year-old children and adults», in Current Psychology Letters, Vol. 24, Issue 2, 2008[En ligne], pages 40-51, published on line on : 8 septembre 2008.

URL : http://cpl.revues.org/document3673.html.

\section{VISUAL/ AUDITORY PROCESSING AND CATEGORIZATION PREFERENCES IN}

\section{5-YEAR-OLD CHILDREN AND ADULTS}

\section{Carole Berger}

Laboratoire de Psychologie et NeuroCognition (LPNC - UMR CNRS 5105) \& Département de Psychologie, Université de Savoie, BP 1104, 73000 Chambéry, France carole.berger@univ-savoie.fr

\section{Sophie Donnadieu}

Laboratoire de Psychologie et NeuroCognition (LPNC - UMR CNRS 5105) \& Département de Psychologie, Université de Savoie, BP 1104, 73000 Chambéry, France 


\begin{abstract}
This research explores adults' and 5 year-old children's use of perceptual and conceptual information for categorizing stimuli defined by visual and auditory cues. A forced-choice categorization task contrasting conceptual (i.e., schema-category) and non conceptual relations was used. Choices for conceptual objects were more frequent in adults than in children. They were also more frequent when alternative comparison objects were perceptually dissimilar (versus similar) to the target. Finally, in children (but not in adults), conceptual and perceptual responding depended on processing constraints. Results were discussed in terms of categorization preferences, depending on both task demands and processing capabilities.
\end{abstract}

Key words: categorization, development, vision, audition

In recent years, a substantial amount of research has been carried out examining the role of eventschemas and of perceptual cues (physical aspects) in category representations. Event-schemas correspond to early conceptual representational systems which are organized spatially and temporally (e.g., an event such as breakfast).

According to some authors (Hudson \& Nelson, 1983), these conceptual representations are in use by 2 years of age and even appear in a very simple form by the age of 9 months (Mandler, 2000). When analyzing the linkages between the perceptual and conceptual organizations, some authors describe independent representational systems in which conceptual cues correspond to re-descriptions of perceptual inputs (Mandler, 2000). Other authors argue that only one kind of categorization process is involved : infants first consider perceptual features. Then, following a process of quantitative enrichment, they go on to consider conceptual features (Quinn \& Eimas, 2000). Importantly, the question of the linkages between the perceptual and conceptual organization have been discussed in recent years especially in researches performed in infants under two years of age. This question was less central and was approached differently when older children were considered. Indeed, inductions tasks analyzed how children generalized new properties as a function of perceptual cues and category relations (Gelman and Markman, 1986 ; 1987). Forced-choice categorization tasks essentially analyzed the selective activation of perceptual and conceptual organization as a function of task or instruction parameters (Deak and Bauer, 1995 ; Golinkoff, Shuff-Bailey, Olgin \& Ruan, 1995).

In a previous study (Berger \& Donnadieu, 2006), we outlined a number of hypotheses concerning the way perceptual and conceptual cues might be linked in childrens' and adults' categorizations. Perceptual cues referred to available physical characteristics of the objects, while conceptual cues referred to previously acquired knowledge. Although this knowledge can cover different aspects, we focused on spatial and temporal representations of objects within a given schema (i.e., schema-based knowledge). The same perceptual and conceptual aspects will be considered in the present study. Berger and Donnadieu (2006) used a forced-choice categorization task in which a target object had to be paired with one of two comparison objects a) from the same schema and $b$ ) from a different schema than the target respectively. By varying the perceptual distance between the targets and comparison objects, the authors analyzed the extent to which schema-based (i.e., conceptual) responding might depend on perceptual factors. This was partially found to be the case in a classic situation using visual stimuli. The authors further examined the possiblity that these results could be explained by sensory parameters or/and processing constraints. This question was investigated by varying both the 
presentation of the stimuli (simultaneous versus sequential) and the sensory modality (vision versus audition). It was concluded that the auditory modality induced a more perceptually constrained processing than the visual modality. Perceptual and conceptual cues also appeared to be more strongly linked in audition than in vision (in audition, selection of schema-based responses depended on all perceptual similarity relations available in a given triad). In addition, the mode of presentation had an influence since perceptual responding increased in young children when the stimuli had to be processed sequentially (versus simultaneously).

In an attempt to explain the origin of the high consideration of perceptual information in audition, we described a "processing limitation" hypothesis. We suggested that the auditory system's difficulties in carrying out an analysis based on conceptual cues could be the consequence of the sequential mode of information processing (which constitutes a fundamental characteristic of audition). Indeed, in previous studies, authors have focused on the temporal factors involved in the processing of objects in order to explain the differential weighting of perceptual and knowledge-based attributes in categorization tasks. For example, Lin and Murphy (2001) proposed that adults' consideration of physical similarities in a visual categorization task could replace that of thematic (schema) relations when the objects are processed in isolation. Rey and Berger (2001) discussed analogous effects in children when lexical learning tasks were used.

Effects due to processing constraints and temporal factors are further investigated in the current study in the context of a bi-modal categorization task allowing participants to shift from auditory to visual cues. Our aim is to determine whether, in such a context, sequential presentation might preclude a mode of categoriztion based on knowledge attributes. Following our initial line of research, we will also examine whether, due to limitations in terms of memory and attentional processes (Pascual-Leone, 1988), specific patterns of responding could be at work in children.

Stimuli defined by visual and auditory cues have been previously used in categorization research preformed on children (Gelman \& Markman, 1986 ; 1987). It should be noted that these studies addressed the question of how properties (i.e., knowledge about a given object) were generalized towards new objects. In these experimental conditions, attention was highly focused on conceptual aspects. Auditory cues were also specific in these studies since they corresponded to verbal labels of objects (whereas visual cues corresponded to pictures of objects). Gelman and Markman $(1986,1987)$ provided evidence of a prominence of conceptual responding in young children. Such findings may well be a consequence of the influence of words when considering conceptual cues (Liu, Golinkoff \& Sak, 2001). In contrast with these results, Berger and Donnadieu (2006) reported some difficulties in performing a comparison based on conceptual features in the auditory mode. It is important to note that auditory cues of a non verbal nature were used in this research. Following this line of investigation, we shall study categorization in response to non verbal stimuli defined by both auditory and visual cues.

A bi-modal context is proposed here in order to minimize the difficulties previously reported in audition when performing a comparison based on conceptual features. This offers the possibility of using visual conceptual cues if the correspondingly auditory cues are not available. It could, consequently, prove useful in clarifying why prominent perceptual responses occurred in children when only audition was involved (Berger \& Donnadieu, 2006). The demand in terms of memory storage and attention is supposed to be relatively low in the bi-modal presentation, due to the possibility of performing an additional visual analysis. Following our hypothesis in terms of processing constraints, perceptual responding would then no longer be prominent. Age-related changes and effects due to the mode of presentation will also help to clarify how processing capabilities and memory/ attentional constraints might influence categorization responding. 
In order to study these issues, we will examine children and adults' preferences in a forced choice categorization task. In each trial, a target and two comparison objects (both from the same taxonomic category as the target) will be presented. The first comparison object (same schema as the target) will be perceptually distant (on visual and auditory cues) from the target. The second comparison object (different schema) will vary according to its perceptual distance (on visual or/and auditory cues) from the target. The aim of the research is to investigate whether the perceptual resemblance of this different schema object reduces the frequency of alternative (i.e., schema-based) responses. This type of effect has already been observed in audition but not in vision (Berger \& Donnadieu, 2006). Could the relationship between perceptual and conceptual cues be enhanced in the present research, which provides the possibility of considering varying types of perceptual features ? If that were the case, what would be the differential weightings of auditory and visual information ? In order to reply to these questions, we studied the performances of 5-year-old children (high consideration of schema relations, Nelson, 1983) and of adults, using different contexts of presentation (sequential versus simultaneous presentation).

\section{METHOD}

\section{PARTICIPANTS}

The participants were eighty children, aged between 5 years 3 months and 6 years 1 month (mean age = 5 years 8 months), and 80 adults. The children were pupils at a preschool in Chamberry and the adults were students at the University of Savoie. Informed consent was obtained from the parents of the children and from the adult participants.

\section{MATERIALS AND PROCEDURE}

Eight stimulus sets (see Table 1) were used, each one including 4 triads. In each triad, one target (for example a plane, see Figure 1) and two comparison objects were presented. Both comparison objects were conceptually related to the target since they were from the same taxonomic (super-ordinate level) category.

Table 1. List of stimulus sets

\begin{tabular}{cccc}
\hline Set & Targets & $\begin{array}{c}\text { "same schema" } \\
\text { (Contiguity) }\end{array}$ & "Different schema" \\
\hline 1. & Plane & Helicopter & Car \\
2. & Drops of rain & Wind/ Storm & Shower (jet of water) \\
3. & Truck & Motorbike & Boat \\
4. & Door bell & Door "toc toc" & Alarm \\
5. & Chimpanzee & Lion & Doggy \\
6. & Clock bell & Church bell & Watch \\
7. & Pig & Cow & Lion \\
8. & Duck & Cock & Seagul \\
\hline
\end{tabular}


Figure 1. Visual representations of the target and comparisons objects used for set 1 .

Depending on the situation, the "different schema" object was visually similar (St. 1) to the target or dissimilar (St. 2).

The typical sound produced by the car was also similar or dissimilar with that produced by the target plane (V+A+ : St. 1 / close motor sound, V+A- : St. 1 / far motor sound, V-A+ : St. 2 / close motor sound, V-A- : St. 2 / far motor sound).

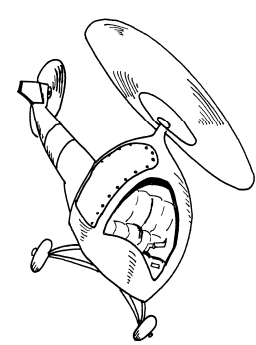

(St. 1)
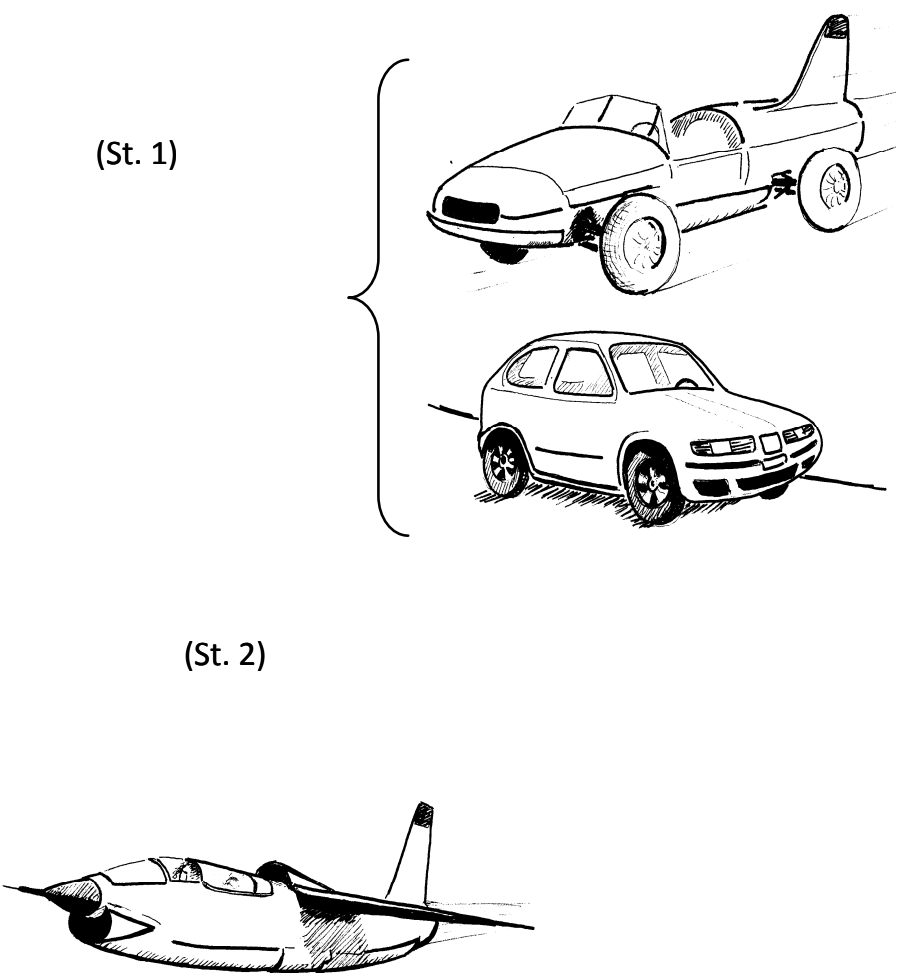

Target 
The first comparison object, also known as the "same schema" object (i.e., a helicopter) had an additional conceptual link with the target, corresponding to a spatial contiguity relation. It was chosen to be perceptually distant from the target on both visual and auditory cues (V. A.). The second comparison object, also known as the "different schema" object (i.e., a car) had no contiguity relation with the target, but could be more or less close to it from a perceptual point of view. Depending on visual and/or auditory perceptual distances, 4 situations were considered :

V. A : visual and auditory perceptual dissimilarities with the target,

$V_{+} A_{+}$: visual and auditory perceptual similarities with the target,

$V_{+} A_{-}$: visual perceptual similarity and auditory perceptual dissimilarity with the target,

$V_{-} A_{+}$: visual perceptual dissimilarity and auditory perceptual similarity with the target.

In each triad, the participants were then given a target object, a "same schema" comparison object perceptually dissimilar to the target (high perceptual distance visually and auditory: $V-A-$ ), a "different schema" comparison object that varied according to its perceptual distance with the target $\left(V_{-} A_{-}, V_{+} A_{+}, V_{+} A_{-}, V_{-} A_{+}\right.$, see below). The 4 triad types used in a given set varied then only according to the characteristics of the "different schema" object.

Participants were informed that they would have to consider different stimuli, each one being represented visually (picture of the object) and by auditory cues (typical sound produced by the object and allowing its identification). They were informed that the target object would appear at the bottom of the screen, in a center position, whereas the comparison objects would appear at the top of the screen (left and right positions). When the target was presented (visual and auditory presentation), the participants were told: "look at and listen to this. Afterwards, you will have to find the item that goes best with it". Then, the first and the second comparison objects appeared, each represented by both visual and auditory cues. In the simultaneous presentation condition, the picture of each object stayed in place until the subject gave his response. The participants were informed that they could listen to each sound as frequently as they wanted (by pointing at the picture). The same procedure was used in the sequential presentation condition, the sole difference being that the picture of each object disappeared following its presentation. It was replaced by a non-sense icon, which had to be presented in order to materialize the position of the stimulus. The participants were informed that they could look at/listen to each picture/sound as frequently as they wanted, by pointing to the stimulus position.

Since the aim of the research was to establish participants' preferences, the "go best" instruction, which allowed either perceptual (visual or/and auditory) or conceptual responses, was used. The triad type had to be treated as a between-subject factor even though this was not the case in our previous work (Berger \& Donnadieu, 2006) where a within-subjects design was used. The choice of design was indeed constrained by the relationship linking targets and comparison objects described previously. In the within subject-design, participants may well have noticed that one of the comparison objects was always the same (in a given set). This could have induced stereotyped responses.

Children and adults were randomly assigned to each of the experimental conditions, defined by the nature of the presentation (sequential, simultaneous) and the type of triad ( $\left.V_{-} A_{,} V_{+} A_{+}, V_{+} A_{-}, V_{-} A_{+}\right)$. Each 
participant was presented with 8 stimulus sets in a random order. Right and left positions for the "same schema" and "different schema" comparison objects were counterbalanced.

\section{PRIOR SIMILARITY RATINGS}

We used MultiDimensional Scaling analysis (MDS) for controlling the perceptual relationships between stimuli (Kruskal, 1964 a, b; Shepard, 1962 a, b). The aim of the MDS analyses was to select, for each stimulus set and in each modality (vision and audition), 2 stimuli highly dissimilar to the target (one from the same schema, the other from a different schema) and one stimulus highly similar to the target (from a different schema).

In the visual test, for a given stimulus set, 10 pictures were chosen corresponding to one target and 9 exemplars. Six of these exemplars were from a different schema than the target ( 3 were chosen to be perceptually similar to the target, 3 were chosen to be perceptually dissimilar). The 3 other exemplars (all chosen to be perceptually dissimilar to the target) were from the same schema than the target. The 10 pictures were presented by pairs. Twenty students at the University of Savoie (mean age $=21$ years-old) were asked to rate the degree of perceptual dissimilarity between the two pictures on a scale varying from 1 (very similar) to 9 (very dissimilar). The perceptual nature of the rating was emphasized during the instructions. A dissimilarity matrix was calculated by averaging the results for all 20 subjects. This was used as input to the STATISTICA multidimensional scaling program to obtain the mean metric distances between targets and comparison objects. The auditory test was performed in a similar way, except that the participants had to rate the similarity between corresponding sounds.

The final constitution of a given set included : a target, a same schema object chosen to have the greatest perceptual distance with the target, and 4 different schema objects respectively chosen to have : a) the greatest perceptual distances with the target $(V-A-), b)$ the smallest perceptual distances $(V+A+), c)$ the smallest visual distance and the greatest auditory distance $(V+A-), d)$ the greatest visual distance and the smallest auditory distance ( $\mathrm{V}-\mathrm{A}+$ ). We ensured that the 6 objects within each set could be easily recognized, both in vision and in audition (more than $90 \%$ of correct recognition for each stimulus in a group of 12 children aged 5-6). The mean metric distances (averaged across the 8 items) between targets and comparison objects for the selected stimuli are presented in Table 2.

Table 2. Mean metric distances (averaged across the 8 items) between targets and comparison objects.

\begin{tabular}{cccc}
\hline Modality & "same schema" & $\begin{array}{c}\text { "Different schema" } \\
\text { High perceptual distances } \\
\text { (dissimilar) }\end{array}$ & $\begin{array}{c}\text { "Different schema" } \\
\text { Low perceptual distances } \\
\text { (similar) }\end{array}$ \\
\hline Visual & 7.5 & 7.2 & 2.9 \\
Auditory & 7.2 & 7.2 & 4.2 \\
\hline
\end{tabular}




\section{RESULTS}

The mean number of schema category responses (i.e., selection of "same schema" comparison objects) is shown in Figure 2 as a function of age and triad type in the simultaneous presentation condition (Figure 2a) and in the sequential presentation condition (Figure 2b).

Figure 2a. Mean number of "same schema" responses as a function of age and triad type in the simultaneous presentation condition.

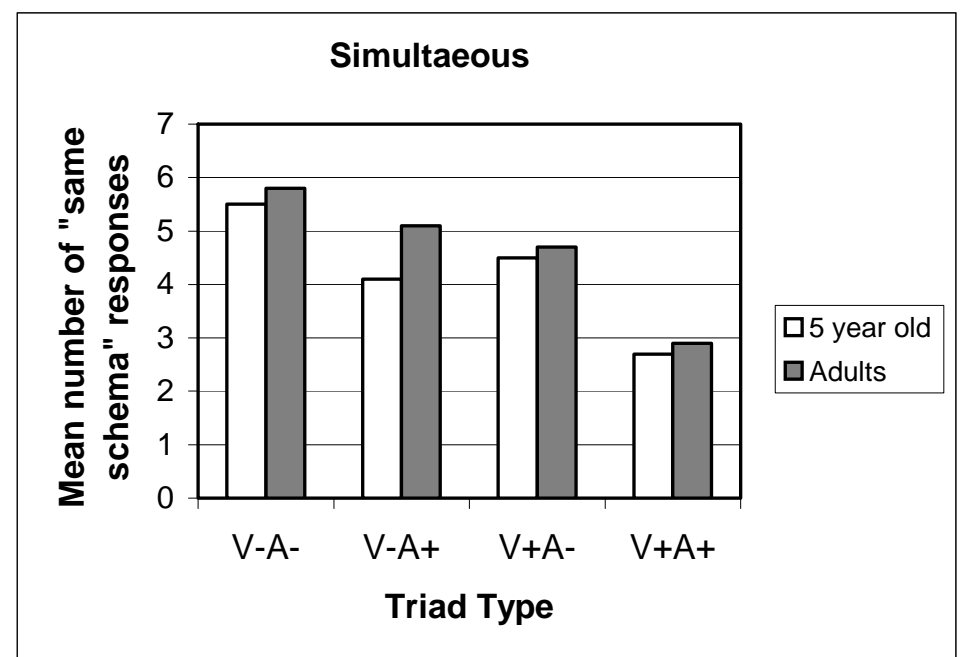

Figure $\mathbf{2 b}$. Mean number of "same schema" responses as a function of age and triad type in the sequential presentation condition.

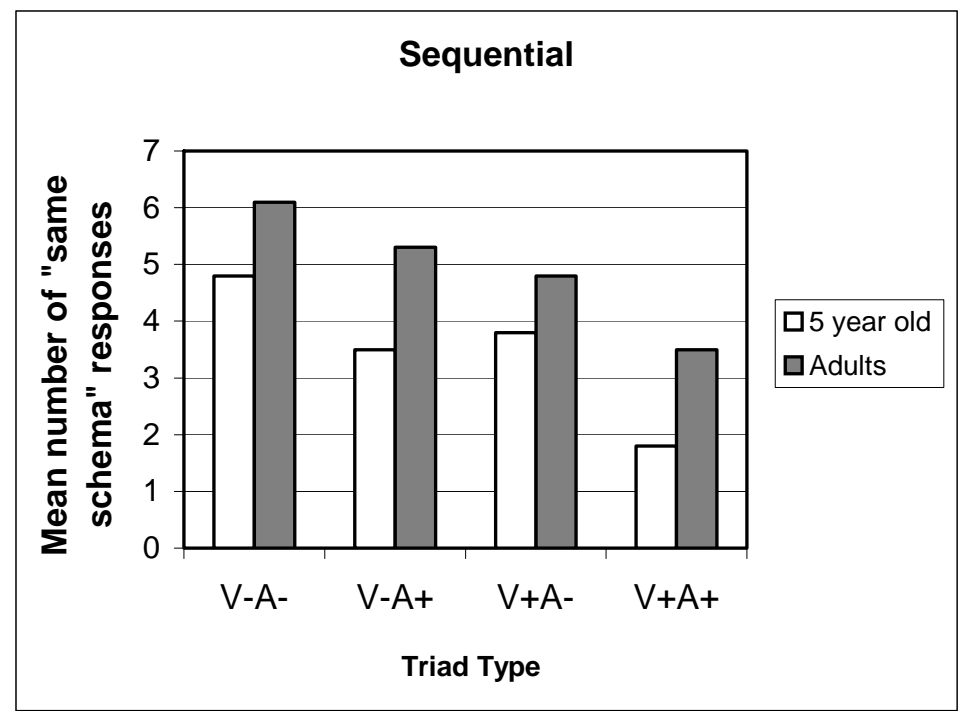


An analysis of variance was conducted on the number of times each participant chose a schema category response. The between-subjects variables were age (5 years old, adult), stimulus presentation (simultaneous, sequential) and triad type (V.A., $\left.V_{+} A_{+}, V_{+} A_{-}, V_{-} A_{+}\right)$. The ANOVA revealed a significant effect of age, $F(1,144)=13.68, p<.001$. Schema category responses were more frequent in adults $(M=4.77)$ than in children $(M=3.83)$. The effect of triad type was also significant, $F(3,144)=21,30, p<.001$. Orthogonal comparisons revealed that schema category responses were less frequent when the "different schema" object was visually or/and auditory similar to the target (i.e., in triad types $V-A+, V+A-, V+A+, M=3.89$ ) rather than when it was dissimilar (i.e., in triad type $\left.V_{-} A_{-}, M=5.55\right), F(1,144)=32.11, p<.001$. Schema category responses were also less frequent when the "different schema" object was similar to the target on both visual and auditory cues (i.e., in triad type $V_{+} A_{+}, M=2.72$ ) rather than when it was similar according to only one kind of perceptual cues (i.e., in triad types $\mathrm{V}-\mathrm{A}+$ and $\mathrm{V}+\mathrm{A}-, \mathrm{M}=4.47), \mathrm{F}(1,144)=31.79, \mathrm{p}<.001$. No difference was observed, however, between visual $(M=4.45)$ and auditory perceptual similarity $(M=4.50)$. The ANOVA also revealed a significant interaction between age and presentation, $F(1,144)=4.09, p<.05$. Specific comparisons showed that the effect of presentation was not significant in adults $(M=4.62$ and $M=4.92$, respectively in the simultaneous and in the sequential presentation conditions). This effect, however, was significant in children, with more schema category responses when a simultaneous presentation was used $(M=4.20)$ than when a sequential presentation was used $(M=3.47), F(1,144)=4.09, p<.05$. No other main effect or interaction was significant.

Additional $t$ tests showed that schema category scores were above chance level only in situations using a perceptually dissimilar alternative object (triad types $\mathrm{V}-\mathrm{A}-$ ). This was the case in children in the simultaneous presentation condition $(t=3.00, p<.02)$ and in adults both in the simultaneous presentation $(t=3.53, p<.01)$ and in the sequential presentation $(t=3.37, p<.01)$ conditions. Finally, schema category scores were below chance level only in children and in situations using a perceptually similar alternative object (triad type $\mathrm{V}+\mathrm{A}+)$. These below chance level scores were observed both in the simultaneous $(t=2.58, p<.05)$ and in the sequential presentation conditions $(t=11.05, p<.001)$.

\section{Discussion}

The experiment presented here examined the consideration of schemas and perceptual information in a bimodal categorization task using objects from the same taxonomic category. Previous research demonstrated that schemas and perceptual cues can help provide conceptual coherence once taxonomic knowledge is available (Lin \& Murphy, 2001). These cues, however, seem to be differently used throughout development. For example, Berger and Donnadieu (2006) reported results showing that schema-based (versus perceptual) relations were more strongly weighted by adults than by children in a forced-choice categorization task. In addition, the nature of the sensory modality seems to play a role since schema responding was highly reduced with auditory processed stimuli, when compared to results observed in vision. Origins of these effects were studied in the present research. By using additional visual cues, we intended to increase the accessibility of conceptual relations. If an explanation in terms of "processing limitation" is relevant, frequent schema responding should be observed in the bimodal context.

Our results confirm the lower consideration of the schema (versus perceptual) structure in children, as compared to adults. This supports our previous observations that children frequently organize their categories according to perceptual features when the auditory modality is involved. It is important to note that they can nevertheless produce prominent schema category responses. This happens to be the case in some contexts of 
the experiment presented here using both auditory and visual cues (i.e., see results on V-A- triads in the simultaneous presentation condition).

Indeed, our bimodal situation offers the possibility of using both perceptual cues (valued by the auditory system) and conceptual cues (valued by the visual system). In the light of results obtained, children appear to favor perceptual responses in the sequential presentation condition and when highly salient perceptual relations are available (in $\mathrm{V}+\mathrm{A}+$ triad types). Schema responses on the other hand, seem to be preferred in contexts having less constraints in terms of attention or memory storage (simultaneous presentation) and when the salience of the perceptual relations is low (in V-A-triad types).

This confirms that the mode of presentation strongly influences categorization responding. Importantly, this effect is demonstrated here in children but not in adults. This is consistent with a "processing limitation" explanation. Since children demonstrate less capabilities than adults especially in terms of memory and attention, their categorization choices could be particularly affected by the mode of presentation. Indeed, comparisons in a categorization task using a sequential presentation have to be performed using stimulus characteristics stored in memory. In these conditions, children prefer to consider perceptual rather than conceptual relations since this helps to reduce the complexity of the situation to a manageable level. In adults, this complexity would be perceived less, due to efficient processing capabilities. Consequently, there would be no need to perform the task from a perceptual point of view.

A second important result of our experiment concerns the relations between the perceptual and conceptual organizations. The different schema object elicited more response choices when it was perceptually close to the target. It should be added that categorization responding was affected in the same way by visual and auditory perceptual distances. Concordant conclusions were obtained when results were analyzed in terms of preferences (i.e., comparisons with chance levels): prominent conceptual (schema-based) responding was at work only if few perceptual similarity relations were available on alternative comparison objects (i.e., triad types V-A-). By contrast, below- chance level scores were observed for conceptual choices in situations offering highly salient perceptual relations (i.e., triad types $\mathrm{V}+\mathrm{A}+$ ).

The substantial and general perceptual effects demonstrated in the current forced-choice categorization task have important implications. Linkages between the perceptual and conceptual organizations were previously shown in the context of an auditory presentation (Berger \& Donnadieu, 2006). We now extend these findings by showing that connections involving the perceptual and conceptual levels of analyses can also have critical roles in the context of a bimodal (auditory plus visual) presentation.

The results reported here are consistent with the recent developmental claim that categorizations based on perceptual factors and those based on conceptual factors do not operate independently (Quinn \& Eimas, 2000). Moreover, they provide information on how children and adults relate perceptual and conceptual attributes in daily-life categorization. The bi-modal context used in the present experiment is interesting from an ecological point of view since many objects are processed by both the visual and auditory sensory modalities in everyday life. Indeed, as vision and audition are adapted in order to access distant characteristics, they play central roles in the perception of objects. Previous research using verbal (MacDonald \& McGurk, 1978) or non verbal (Saldana \& Rosenblum, 1993) stimuli clearly demonstrated that complementary information is provided by the visual and auditory systems. The correspondences between the perceptual and conceptual levels of analysis evidenced in the visual/ auditory context of our experiment is consistent with this view. It should be noted that analogous correspondences were reported in other forced-choice categorization tasks using both auditory and visual cues. This was the case, for example, in research studying lexical learning. It was proposed that perceptual information would help children considering the taxonomic organization when learning new words (Imai \& al., 1994). 
Finally, in addition to previously established findings, our results enable us to insist on the importance of contextual factor for describing the nature of categorization skills. Previous studies have examined the relative valuations of perceptual and of conceptual attributes as a function of different situational factors such as labeling (Baldwin, 1992 ; Golinkoff, Shuff-Bailey, Olgin \& Ruan, 1995; Imai, Gentner \& Uchida, 1994), instructions (Bonthoux \& Berger, 2001; Deak \& Bauer, 1995), stimulus characteristics (such as pictures or physical objects, Liu, Golinkoff \& Sak, 2001) or presentation constraints (Rey \& Berger, 2001, Berger \& Donnadieu, 2006), etc. It should be noted that the influences of these different factors have always been explored using visually processed stimuli. We now have data suggesting that the sensory characteristics of the situation could also have an impact on how individuals weigh available relations in a categorization task. In vision, schema relations were described as being salient and meaningful for categorizing taxonomically related objects (Lin \& Murphy, 2001). Previously, we provided evidence that consideration of these relations was reduced when taxonomically related objects were processed using auditory cues, rather than visual cues (Berger \& Donnadieu, 2006). We now demonstrate, in a the context of a bi-modal (auditory/visual) experiment, that schema-based responding needs to be analyzed as a function of both stimulus perceptual characteristics and stimulus presentation constraints.

In conclusion, the fact that response choices can result from an interaction between the perceptual and conceptual levels of analyzes is important for future research in categorization. Effects due to the roles of perceptual distance and of temporal factors (see also Berger \& Hatwell, 1993) suggest that the availability of attributes, in relation with processing capabilities, certainly plays a central role in this interaction. The problem lies not in determining what types of categories (perceptual versus conceptual) are accessible at a given developmental level in daily life, but rather, in analyzing how multiple situational or individual factors operate to increase or reduce the salience of each available information. In a previous study (Berger \& Donnadieu, 2006), we concluded that the consideration of schema (versus perceptual) relations was highly reduced in audition, in comparison to vision. We now report results showing that an audio-visual presentation is compatible with an analysis of both conceptual and perceptual attributes. Future research will be necessary in order to establish whether the presence of additional visual cues could be responsible for these changes in responding. In this respect, forced-choice categorization tasks allowing direct comparisons between bi-modal and uni-modal perceptual contexts will have to be used. Following the line of investigation developed in the current paper, results will also have to be analyzed as a function of developmental level, perceptual similarity and stimulus presentation. Emphasizing the respective influence of these factors in different sensory contexts will help to understand the origin of forced-choice categorization decisions.

\section{REFERENCES}

Baldwin, D. A.(1992). Clarifying the role of shape in children's taxonomic assumptions. Journal of Experimental Child Psychology, 54, 392-416.

Berger, C. \& Donnadieu, S. (2006). Categorization by schema relations and perceptual similarity in 5-year-olds and adults : A study in vision and in audition. Journal of Experimental Child Psychology, 93, 304-321.

Berger, C. \& Hatwell, Y. (1993). Dimensional and overall similarity classifications in haptics: a developmental study. Cognitive Development, 8, 495-516.

Bonthoux, F., \& Berger, C. (2001). Role of perceptual and conceptual properties in 5-year-olds' reasoning: categorization tasks with instruction based on objects or object properties. Current Psychology Letters/Behavior, Brain \& Cognition, 3 (6), 27-40. 
Deák, G. O., \& Bauer, P. J. (1995). The effect of task comprehension on preschoolers and adults' categorization choices. Journal of Experimental Child Psychology, 60, 393-427.

Gelman, S.A., \& Markman, E.M. (1986) Categories and induction in young children, Cognition, 23, 183-209.

Gelman, S.A., \& Markman, E.M. (1987) Young children's inductions from natural kinds: The role of categories and appearances. Child development, 58, 1532-1541.

Golinkoff, R. M., Shuff-Bailey, M., Olgin, R., \& Ruan, W. (1995). Young children extend novel words at the basic level: Evidence for the principle of categorical scope. Developmental Psychology, 31, 497-507.

Hudson, J., \& Nelson, K. (1983). Effect of script structure on children's story recall. Developmental Psychology, $19,625-635$.

Imai, M., Gentner, D., \& Uchida, N. (1994). Children's theories of word meaning: the role of shape similarity in early acquisition, Cognitive Development, 9, 45-75.

Kruskal, J.B. (1964a). Multidimensional scaling by optimizing goodness of fit to a nonmetric hypothesis. Psychometrika, 29, 1-28.

Kruskal, J.B. (1964b). Non-metric multidimensional scaling: A numerical method. Psychometrika, 29, $115-129$.

Lin, E. L., \& Murphy, G. L. (2001). Thematic relations in adults' concepts. Journal of Experimental Psychology: General, 130, 3-28.

Liu, J., Golinkoff, R.M., \& Sak, K. (2001). One cow does not an animal make : young children can extend novel words at the superordinate level. Child Development, 72, 1674-1694.

MacDonald, J. \& McGurk, H. (1978). Visual influences on speech perception processes. Perception and Psychophysics, 24, 253-257.

Mandler, J. M. (2000). Perceptual and conceptual processes in infancy. Journal of Cognition and Development, 1, 3-36.

Nelson, K. (1983). The derivation of concepts and categories from event representations. In E.K. Scholnick (Ed.), New trends in conceptual representation: Challenges to Piaget's theory? (pp129-149). Hillsdale, NJ: Erlbaum.

Pascual-Léone, J. (1988). Organismic processes for neo-Piagetian theories: a dialectical causal account of cognitive development, in A. Demetriou (ed.), The neo-Piagetian theories of cognitive development : Toward an integration (p. 25-64). Amsterdam, North-Holland.

Quinn, P. C., \& Eimas, P. D. (2000). The emergence of category representations during infancy: Are separate perceptual and conceptual processes required? Journal of Cognition and Development, 1, 55-61.

Rey, E. \& Berger, C. (2001). Four- and five-year-old children's categorization; sensitivity to constraints on word meaning and influence of stimulus presentation in a forced-choice paradigm. Current Psychology of Cognition, 20, 63-85.

Saldana, H.M., \& et Rosenbloom, L.D. (1993). Timbre cues and the identification of musical instruments. Journal of the Acoustical Society of America, 36, 2021-2026.

Shepard, R.N. (1962a). The analysis of proximities: Multidimentional scaling with an unknown distance function. Part I. Psychometrika, 27, 125-140.

Shepard, R.N. (1962b). The analysis of proximities: Multidimentional scaling with an unknown distance function. Part II. Psychometrika, 27, 219-246. 